\title{
Quantitation in inflammatory pleural disease to distinguish tuberculous and paramalignant from chronic non-specific pleuritis
}

Surgical Pathology Division of the

Hospital das Clínicas,

University of São

Paulo School of

Medicine, São Paulo,

Brazil

V L Capelozzi

P H N Saldiva

L Antonângelo

T S de Carvalho

Â Logulo

Pulmonary Division

C R R de Carvalho

D Deheinzelin

Correspondence to:

Dr Capelozzi, Departamento

de Patologia, Faculdade de

Medicina da USP, Av. Dr.

Arnaldo 455, CEP

01246-009, São Paulo, SP,

Brasil.

Accepted for publication

15 July 1997

Vera Luiza Capelozzi, Paulo Hilário N Saldiva, Leila Antonângelo, Teóclito Sachetto de Carvalho, Ângela Logulo, Carlos Roberto Ribeiro de Carvalho, Daniel Deheinzelin

\begin{abstract}
Aim-To determine by morphometry if pleural biopsies with the histopathological diagnosis of "non-specific pleuritis", malignant, and tuberculous disease could be distinguished morphologically from those with truly non-specific disease.
\end{abstract}

Methods-Each pleural biopsy was reviewed taking into account three compartments of reference: the visceral/parietal mesothelial compartment, the submesothelial screen compartment, and the submesothelial adipose tissue compartment. Normal connective tissue, granulation tissue, fibrocellular proliferation, fibrin, polymorphonuclear cells, mononuclear cells, and mesothelial cells were measured using conventional point counting procedures in terms of the fractional area occupied by each parameter within each compartment of reference. Ranking was carried out on 164 patients, based on their diagnosis: chronic non-specific disease ( $n=57)$, tuberculosis $(n=27)$, malignant disease $(n=58)$, and conditions associated with transudative effusions $(n=22)$. Results-Stepwise discriminant analysis of the resulting data showed that biopsies from patients with tuberculosis, malignant disease, and chronic non-specific disease could be distinguished between themselves and normal cases. Statistical differences among the four groups were observed for eight morphometric parameters related to components of inflammation and extension throughout the three pleural anatomical compartments. A robust discriminant function permitted an adequate classification of the three groups of disease in $88.41 \%$ of the cases. Pleural biopsies with fibrin incorporated within granulation tissue on the submesothelial screen compartment showed $100 \%$ specificity for patients with tuberculosis, while mononuclear cells in a band-like infiltrate on the submesothelial adipose tissue compartment showed $93.1 \%$ specificity for patients with malignant disease. The truly non-specific pleuritis was characterised by deposits of fibrin in the subpleural compartment and discrete signs of chronic inflammation and reparatory fibrosis on the submesothelial screen.
Conclusions-Morphometric analysis of pleural biopsies may be a useful supplementary histological procedure to support the diagnosis of pleural tuberculosis and malignant disease.

(F Clin Pathol 1997;50:935-940)

Keywords: morphometry; pleural biopsy; chronic nonspecific pleuritis

The role of needle biopsy in the evaluation of unexplained pleural effusions is well defined. ${ }^{1-6}$ Histological demonstration of malignancy or granulomatous pleuritis is diagnostic, but the significance of a biopsy that reveals only non-specific chronic pleuritis remains unclear. In a non-specific or normal biopsy specimen there is a $77 \%$ chance that the disease is not present. ${ }^{7}$ However, tumour or tuberculosis may be diagnosed eventually in as many as $40 \%$ of those individuals with an initial biopsy showing chronic non-specific pleuritis. ${ }^{2}$ Repeated pleural biopsies (closed or open) will increase the diagnostic yield in patients proven ultimately to have cancer or granulomatous pleuritis, ${ }^{1-57}$ but will also add to the expense and morbidity of those with chronic non-specific pleuritis. Clinical data may aid in the decision of which patients should be submitted for further diagnostic procedures. However, this approach will still leave some patients underdiagnosed. ${ }^{8}$

There are very few studies of detailed pathological analysis of non-specific histological findings in pleural biopsy specimens. ${ }^{9-11}$ Most previous reports have provided few and subjective criteria for the histopathological interpretation of the pleura.

The purpose of this study was to determine whether morphometric analysis of pleural biopsies (in which a definite diagnosis cannot be reached based upon conventional histopathological aspects) could be useful in the diagnosis of pleural tuberculosis and malignant disease.

\section{Methods}

CLINICAL DATA

All consecutive patients submitted to a closed needle pleural biopsy from 1991 to 1994 at the pulmonary division of the Hospital das Clinicas of the University of São Paulo whose chest roentgenograms and medical records were available for review $(n=311)$ were included in this study. 


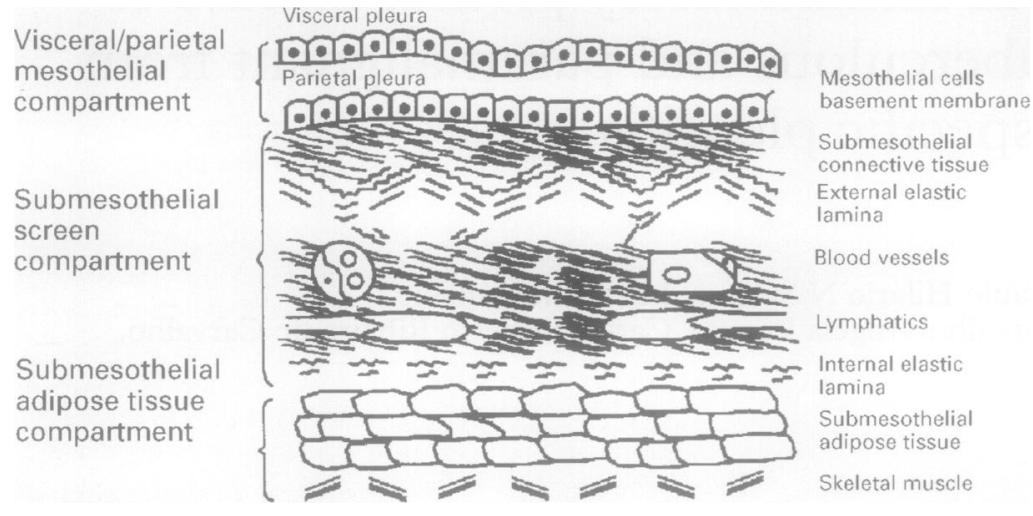

Figure 1 The anatomical layers of the pleura.

The biopsies were performed by a resident or fellow under staff supervision. Three or four specimens of tissue, obtained with a Cope needle, were used for histological study, mycobacterial culture, and fungal culture. Pleural fluid obtained at the time of the biopsy was submitted routinely for a cell count with white cell differential, protein, glucose, and lactate dehydrogenase measurements, bacterial culture, and cytological examination. A second biopsy was performed whenever the pleural fluid exhibited exudative characteristics and the histological findings were non-diagnostic.

In 147 patients, biopsy led to a specific diagnosis. In the remaining 164 patients, chronic non-specific pleuritis was diagnosed after the first closed pleural biopsy and these patients were classified into four groups according to their follow up diagnosis.

Tuberculous disease was the final diagnosis in 27 patients. In nine cases, culture of the
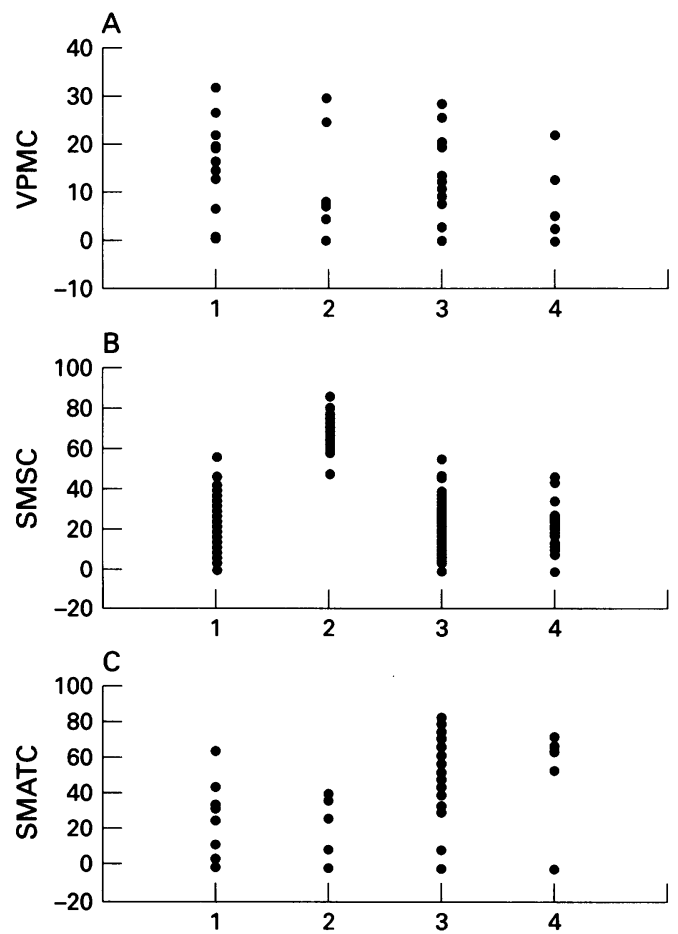

Figure 2 Fractional area (\%) of pleural anatomical compartments expressed as (A) visceral/parietal mesothelial compartment (VPMC), (B) submesothelial screen compartment (SMSC), and (C) submesothelial adipose tissue compartment (SMATC) in the four groups. pleural fragment yielded mycobacteria. In another nine patients a second biopsy disclosed granulomata and pointed towards the diagnosis. In six cases, both the second biopsy and culture of the first biopsy fragment were diagnostic. Three patients were diagnosed based upon sputum isolation of mycobacteria.

Patients were considered to be bearers of malignant disease $(n=58)$ when neoplasm was found with no other identifiable cause for an exudative effusion. Diagnosis was reached by a second pleural biopsy in nine cases, by cytological analysis of the pleural fluid in 11 cases, and by a second biopsy and cytological examination in eight cases. In the remaining 30 cases, thoracic malignant disease was found by means of other diagnostic procedures such as bronchoscopy, mediastinal or transthoracic biopsy, or the discovery of carcinoma at another site such as the breast. In these cases, malignant cells were not found in pleural tissue or fluid, rendering the effusions as paramalignant (associated with malignancy but with no pleural involvement).

After at least three months of follow up subsequent to the second biopsy, 57 patients with exudative pleural effusion showed no evidence of malignancy or tuberculous disease and had a clinical diagnosis (atypical pneumonia, parapneumonic effusions, hepatitis, and splenic infarct) compatible with chronic non-specific disease. This group was classified as chronic pleuritis. Although a follow up of three months is somewhat short, it is of significance when malignancy and/or tuberculosis are considered.

Patients with clinical conditions associated with transudative effusions (congestive heart failure, cirrhosis of the liver, and nephrotic syndrome) and whose follow up of at least three months showed no evidence of malignancy or tuberculous disease composed the fourth group $(n=22)$.

\section{MORPHOLOGICAL STUDY}

The same slides containing biopsy specimens in cubes or strips $2-3 \mathrm{~mm}$ long, analysed for the initial pathological report (paraffin embedded, haematoxylin and eosin stained) were reviewed blind until the final diagnosis.

Morphometry was performed on the different layers of the pleura. The anatomical layers of the pleura are shown diagrammatically in fig 1. Histologically, the parietal pleura is composed of two different layers: a complete layer of mesothelial cells and a thin screen of submesothelial connective tissue, with a well developed network of collagenic and elastic fibres, which contains lymphatic and blood vessels. This layer is in continuity with one composed of adipose and skeletal muscle tissues. The tissues in the pleural biopsies were divided into three compartments of reference: (1) the space between the parietal and visceral pleura was designated as the visceral/parietal mesothelial compartment; (2) the mesothelium and the thin submesothelial conjunctive tissue was designated as the submesothelial screen compartment; and (3) the continuous layer of adipose tissue underlying the submesothelial 

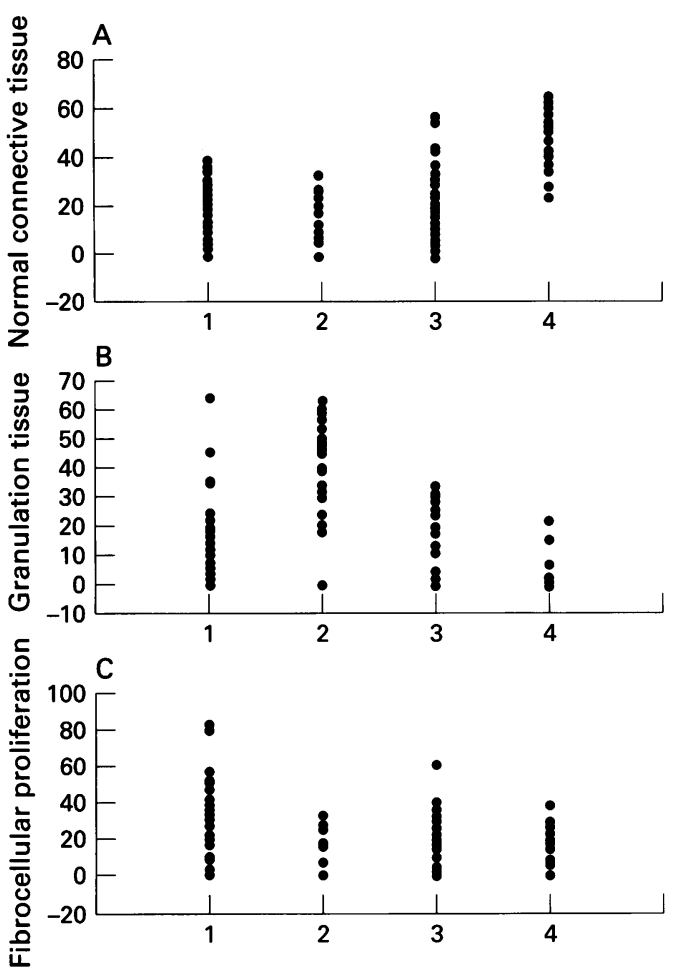

Figure 3 Fractional area (\%) of tissue types expressed as (A) normal connective tissue, (B) granulation tissue, and (C) fibrocellular proliferation in the four groups.

screen was designated as the submesothelial adipose tissue compartment.

Subsequently, specimens were evaluated for: (1) tissue type (normal connective tissue, granulation tissue, and fibrocellular proliferation); (2) components of inflammation (fibrin, polymorphonuclear cells, and mononuclear cells); (3) mesothelial cells; and (4) extension and distribution of the pathological process throughout the three anatomical compartments.

Morphometric studies were performed by a conventional point counting procedure, ${ }^{12}$ using a reticulated eyepiece (100 points and 50 lines). Counting was performed using a cascade progressive sampling approach. In each case, at a magnification of $\times 100$, five noncoincident microscopic fields were studied to quantify the relative fraction of the three pleu-
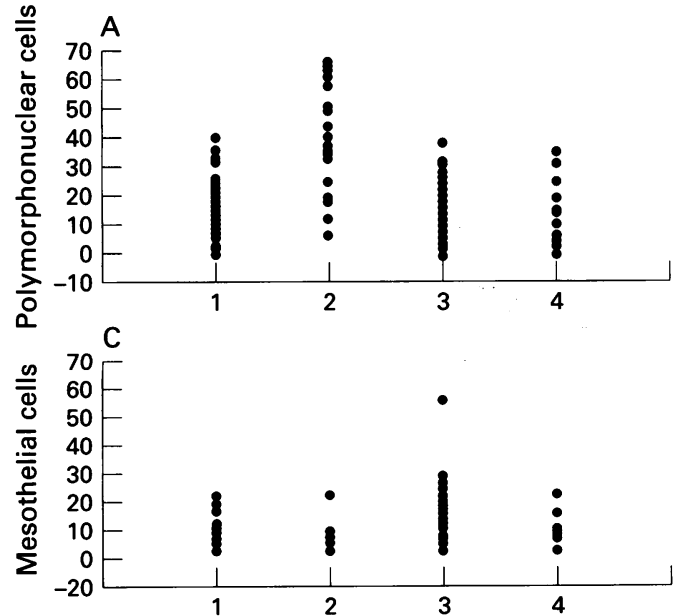

ral compartments by counting a total of 500 points that covered an area of $1 \mathrm{~mm}^{2}$ per biopsy. The error of this procedure was estimated according to Gundersen ${ }^{12}$ and was smaller than $5 \%$. Next, the numbers of points overlying fibrin, granulation tissue, and fibrocellular proliferation were counted in five fields, at a magnification of $\times 400$, covering an area of $62500 \mu \mathrm{m}^{2}$ in each pleural compartment, actually representing the fractional area occupied by each parameter within each compartment of reference. Finally, five fields, at a magnification of $\times 1000\left(10000 \mu \mathrm{m}^{2}\right)$ were counted in each compartment to evaluate the fractional area occupied by polymorphonuclear cells (neutrophils + eosinophils), mononuclear cells (macrophages + lymphocytes + plasma cells), and mesothelial cells. The relative fraction of area was obtained by dividing the values reached by the corresponding parameter under study. Interobserver and intraobserver variability was $10 \%$ and $8 \%$, respectively.

\section{STATISTICAL ANALYSIS}

Discriminant analysis was used to obtain a statistical classification of the four groups. This method finds the linear (additive) combination of variables that gives the clearest separation from individuals into different groups. This procedure includes identification of the variables that contribute significantly to discrimination. The criterion for inclusion of a variable was an $F$ value of 3.0 or more, roughly corresponding to $\mathrm{p}=0.04$. Further independent combinations of the same variables were calculated. Plotting of the values from the first two linear discriminant functions combined for each individual, showed almost the separation achieved between the groups. A stepwise procedure was used to select the variables relevant to distinguish the groups. Because the discriminant power would be optimistic when assessed on the same data used to derive the functions, a jack knife (one out) procedure was included in the results. This procedure withdraws one patient (patient 1 , for instance) from the analysis, then the model is re-estimated excluding that patient. Afterwards, the
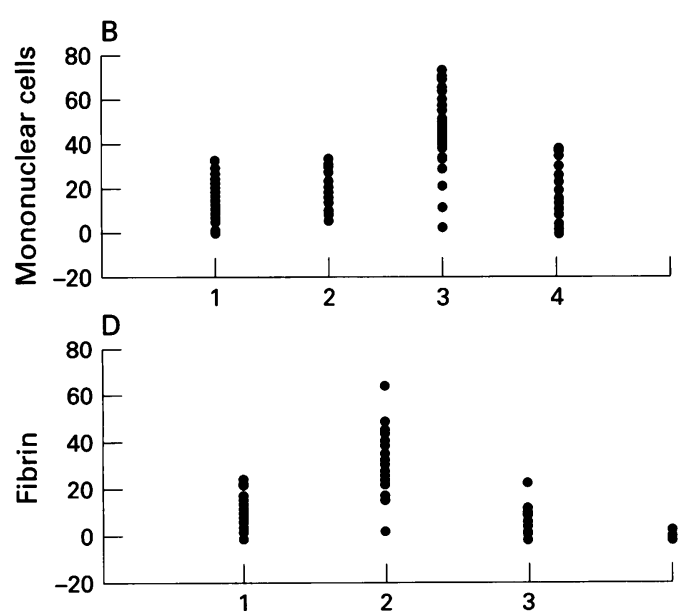

Figure 4 Fractional area (\%) of components of inflammation and mesothelial reactivity expressed as (A) polymorphonuclear cells, (B) mononuclear cells (lymphocytes + plasma cells), (C) mesothelial cells, and (D) fibrin in the four groups. 


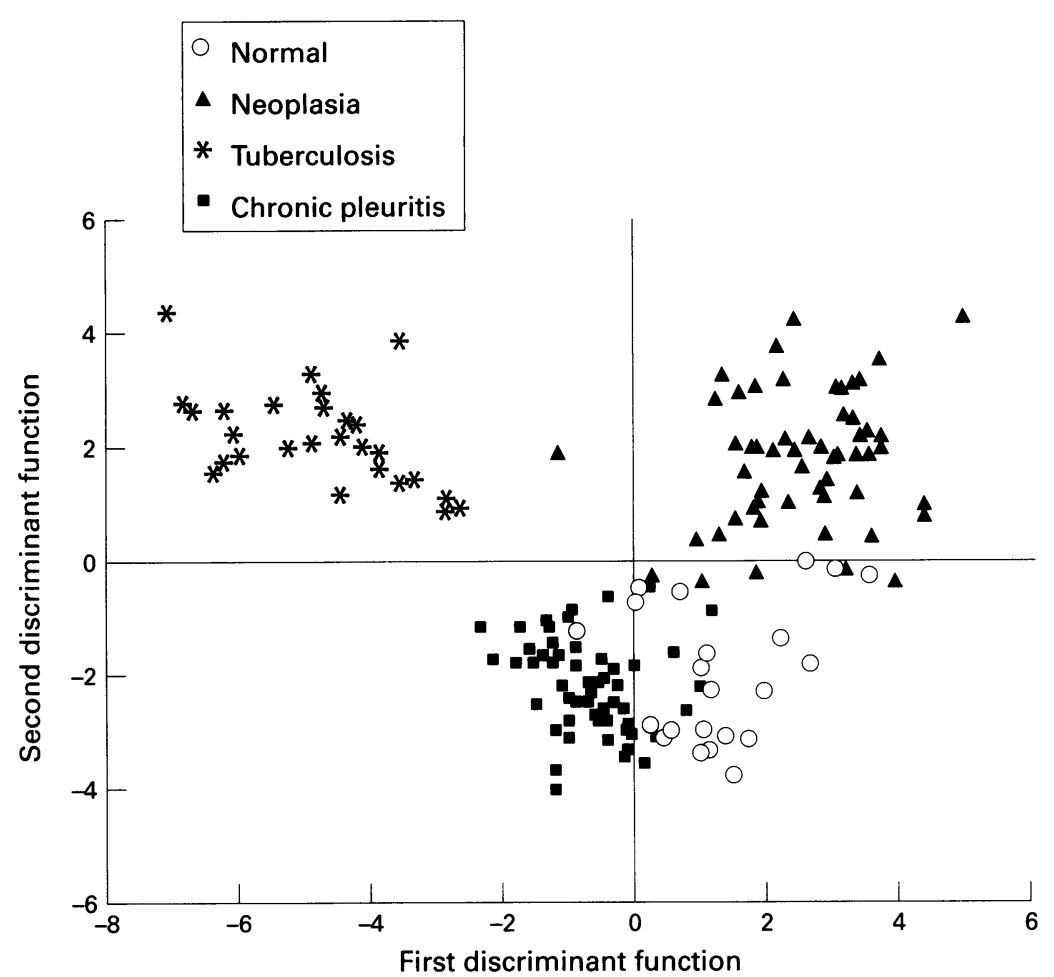

Figure 5 Graphical representation of the results of stepwise discriminant analysis.

excluded patient is classified according to the new model, and his actual classification compared to the predicted one. Next, patient 1 is again included in the analysis and patient 2 is withdrawn, according to the same procedure, until calculations are completed for all patients included in this study. All statistical procedures were performed using the SPPS ( $v 6.0$ ) statistical package $^{13}$ and the level of significance was $0.5 \%$.

\section{Results}

INDIVIDUAL MORPHOMETRIC MEASUREMENTS

Figures 2-4 show the the morphometric data for the 164 serial biopsies. The submesothelial screen compartment (fig 2B), fibrin (fig 4D), granulation tissue (fig 3B), and polymorphonuclear cells (fig 4A) were higher in tuberculosis, whereas the submesothelial adipose tissue compartment (fig 2C) and mononuclear cells (fig 4B) were found more frequently in the group with neoplasia. Fibrocellular proliferation (fig 3C) and normal connective tissue (fig 3A) characterised chronic pleuritis and normal pleura, respectively.

\section{STATISTICAL ANALYSIS}

Using discriminant analysis, different combinations of the morphometric data selected eight variables capable of distinguishing the groups. These are shown below, with a $\mathrm{p}$ value relating to removal from the model: (1) submesothelial adipose tissue compartment, $\mathrm{p}<0.001$; (2) submesothelial screen compartment, $\mathrm{p}<0.001$; (3) visceral/parietal mesothelial compartment, $p<0.001$; (4) fibrin, $\mathrm{p}<0.001$; (5) granulation tissue, $\mathrm{p}<0.001$; (6) fibrocellular proliferation, $\mathrm{p}<0.001$; (7) polymorphonuclear cells, p $<0.001$; and (8) mononuclear cells, $\mathrm{p}<0.001$. These relevant variables were used to construct the

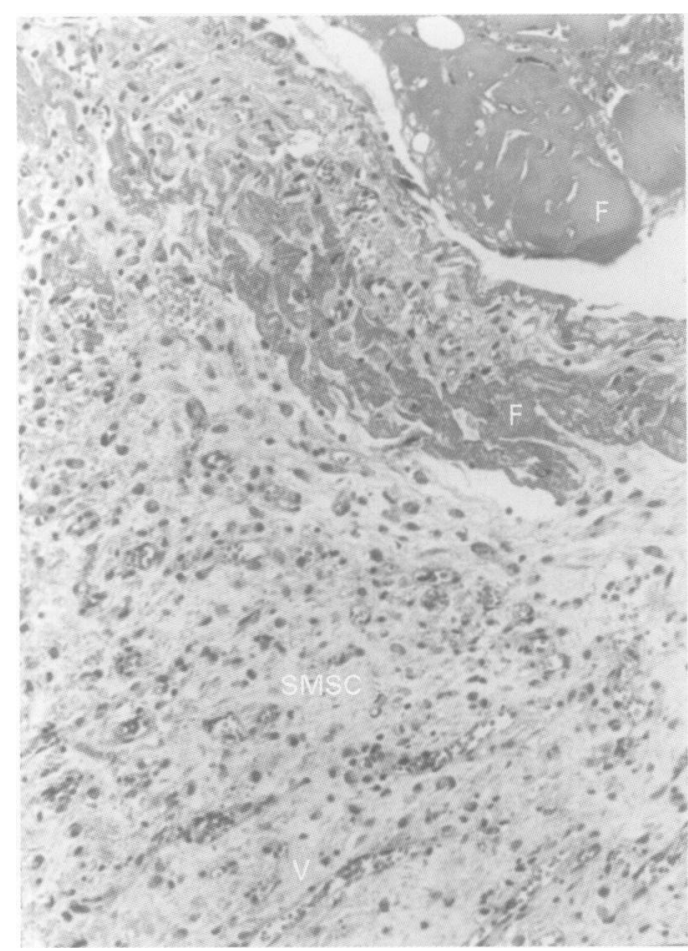

Figure 6 Tuberculous pleuritis: a fibrinous inflammation $(F)$ is the typical morphological reaction seen in the submesothelial screen compartment (SMSC). It is characterised by exudation of plasma proteins and precipitation of masses of fibrin, forming a tangled eosinophilic meshwork to which neutrophils and macrophages are attached. In the botton of this fibrinous deposits the exudate is organised into vascularised connective tissue $(V)$ (haematoxylin and eosin;original magnification $\times 63$ ).

model presented in fig 5, where a plot of the values of the first two linear discriminant functions for each individual shows almost the separation into the groups. Discriminant analysis allowed us to define four distinct patterns of pleural involvement.

Tuberculous pleuritis - the fraction of the area occupied by the submesothelial screen compartment was significantly higher than in the other conditions (fig 2B) and the morphometric quantification of polymorphonuclear cells (fig 4B), fibrin (fig 4A), and vascularised connective tissue (fig 3B) was statistically higher in tuberculosis than in the other groups. Fibrinous inflammation was the typical morphological reaction and this exudate was incorporated into the submesothelial screen, resulting in vascularised connective tissue (fig 6). Twenty seven tuberculous patients $(100 \%)$ were correctly classified as having tuberculous pleuritis (table 1).

Paramalignant pleuritis - the fraction of the area occupied by the submesothelial adipose tissue compartment (fig 2C), mononuclear (fig 4C), and mesothelial cells (fig 4D) was statistically higher in malignant or paramalignant pleuritis than in tuberculous or chronic pleuritis. Here, the submesothelial screen was normal or showed non-specific findings, such as clusters of lymphocytes around the vessels. The submesothelial adipose tissue was infiltrated characteristically by lymphocytes and plasma cells in a band-like pattern (fig 7). Eosinophils were scattered throughout the mononuclear cells. Mesothelial hyperplasia 
Table 1 Percentages of biopsies that were correctly classified by the model (incorporating jack-knife)

\begin{tabular}{llllll}
\hline \multicolumn{5}{c}{ Predicted group membership } \\
\cline { 3 - 6 } Actual groups & $\begin{array}{l}\text { Number of } \\
\text { cases }\end{array}$ & $\begin{array}{l}\text { Chronic } \\
\text { pleuritis }\end{array}$ & Tuberculosis & Neoplasia & Normal \\
\hline Chronic pleuritis & 57 & $48(84.2 \%)$ & $0(0 \%)$ & $0(0 \%)$ & $9(15.8 \%)$ \\
Tuberculosis & 27 & $0(0 \%)$ & $27(100 \%)$ & $0(0 \%)$ & $0(0 \%)$ \\
Neoplasia & 58 & $1(1.7 \%)$ & $0(0 \%)$ & $54(93.1 \%)$ & $3(5.2 \%)$ \\
Normal & 22 & $3(13.6 \%)$ & $0(0 \%)$ & $3(13.6 \%)$ & $16(72.7 \%)$ \\
Total & $164(88.41 \%)$ & & & \\
& & &
\end{tabular}

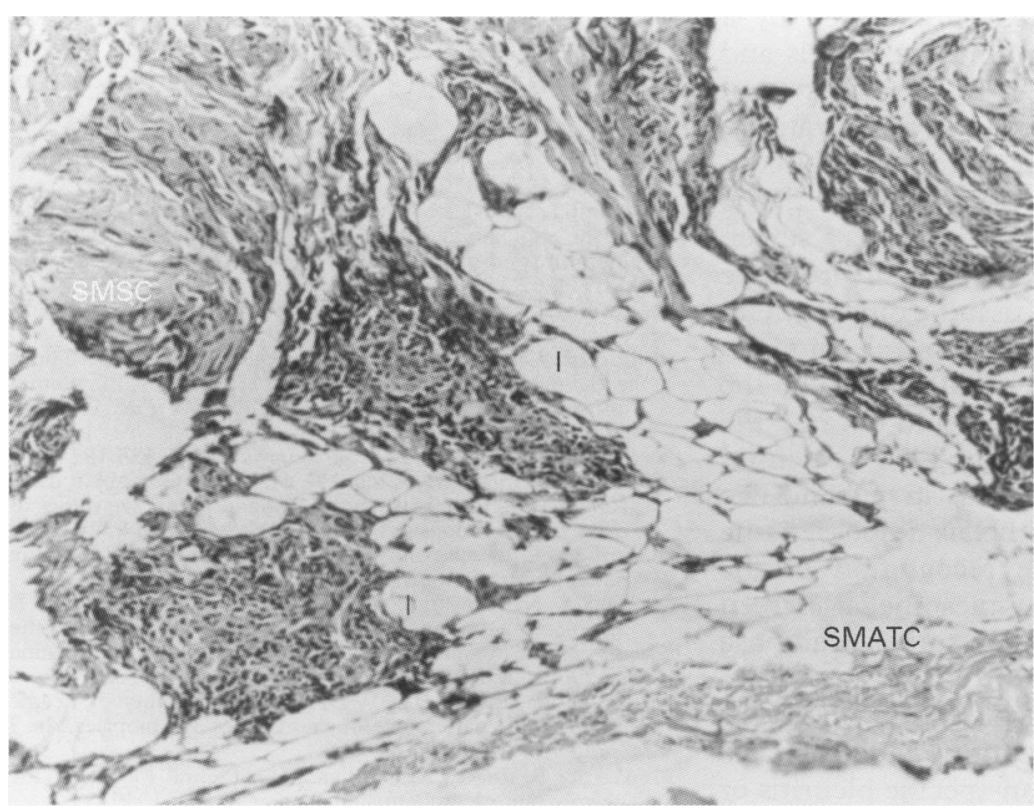

Figure 7 Paraneoplastic pleuritis: characteristically, the submesothelial adipose tissue compartment (SMATC) is infiltrated by lymphocytes, plasma cells, and eosinophils in a band-like pattern (I). Mesothelial hyperplasia is seen re-covering the adipose tissue. Note that the submesothelial screen compartment (SMSC) is normal or shows small clusters of lymphocytes around the vessels (haematoxylin and eosin; original magnification $\times 63$ ).

was observed re-covering the adipose tissue. Fifty four $(93.1 \%)$ of the 58 patients with malignant disease elsewhere' were correctly classified into the group of pleuritis associated with tumour. Three patients from this group were misclassified (table 1) and fell into the group of "transudative effusions".

Chronic pleuritis-the fraction of the area occupied by fibrocellular proliferation (fig 3C) was the morphometric parameter statistically associated with chronic pleuritis. Reviewing these pleural biopsies we could see the true non-specific histological findings: deposits of detached fibrin in the visceral/parietal mesothelial compartment, discrete signs of chronic inflammation (mononuclear cells around the vessels), and reparative thin fibrosis of the submesothelial screen compartment. Normal pleura-the fraction of the area occupied by normal connective tissue (fig 3A) was significantly higher in pleural biopsies associated with transudative effusions, thus characterising pleural involvement in the fourth group.

\section{Discussion}

In this study, we have shown that by means of a simple and quick method, with no additional costs to the patient, it is possible to characterise non-specific pleuritis further and to suggest a specific diagnosis with reasonable accuracy.
The analysis of the results allowed us to identify four distinct patterns of histological pleural involvement.

Tuberculous pleuritis was characterised by a fibrinous inflammation of the submesothelial screen compartment in an absence of granulomata. Our results show how fibrin becomes incorporated into the submesothelial screen, an event that may precede the development of the granumalomatous reaction. Pathologists experienced in reviewing pleural biopsies may believe that these results are not realistic. Indeed, fibrin is associated with most effusions (at least in biopsies), but Paterson's ${ }^{14}$ experimental model for the development of tuberculous pleuritis, which correlates well with what is seen in clinical practice, ${ }^{15}$ supports our findings. Both described how the pleura was replaced extensively by a layer of granulation tissue in which tuberculous granulomas were dispersed. These findings could explain those specimens where the needle reached the tissue between the granulomas and only granulation tissue was found. Another important point is that even in the absence of an intact mesothelial lining, pathologists can distinguish between fibrin in granulation tissue in the submesothelial screen compartment (specific for tuberculosis) and fibrin in the visceral/parietal mesothelial compartment (specific for truly nonspecific pleuritis). In truly non-specific pleuritis the fibrin lies in the visceral/parietal compartment as a detached pseudomembrane, which has not been incorporated into the submesothelial screen compartment. This compartment shows very discrete signs of chronic inflammation and reparative fibrocelllular proliferation instead of fibrinous inflammation.

Paramalignant pleuritis, characterised by a band-like inflammatory reaction of the submesothelial adipose tissue compartment, was the typical morphological reaction seen in pleural biopsies of patients with malignant conditions, but without malignant cells in the pleural tissue at the time of biopsy. Paramalignant pleuritis may be caused by different malignancy associated conditions that do not result from direct pleural involvement. ${ }^{1617}$ The inflammatory changes in the submesothelial adipose tissue compartment might be an immunological reaction of the pleura to tumour produced antibodies. A similar situation occurs in the post-cardiac injury syndrome,$^{18}$ in which an autoimmune phenomenon produces antibodies, causing an exuberant pleuropericarditis rich in mononuclear cells. Exuberant mononuclear infiltrates are a well documented immulogical phenomenon characterising peripheral reactions to tumours, such as gastric cancer. ${ }^{19}$ Also, this band-like cellular infiltrate on the submesothelial adipose tissue might be the result of lymphatic obstruction by tumours. A similar band-like infiltration by mononucleated cells in the submesothelial adipose tissue compartment (associated with non-tuberculous pleuritis) was found by Nagata et al. ${ }^{9}$ Despite the diversity of events that occur in tumour pleural relationships, the present study indicated that a band-like inflammatory reaction is a common feature of cases with paraneoplastic 
involvement. Therefore, this lymphoproliferative response of the pleura to a distant tumour may point to the need for a thoracoscopy or other diagnostic procedure.

Pathologists would argue that chronic inflammation and its sequelae could interfere with the recognition of the border between the submesothelial screen compartment and the submesothelial adipose tissue compartment. In this study, we felt that chronic inflammation and its sequelae in the submesothelial adipose tissue compartment were characterised by a mononuclear infiltrate around the vessels and replacement of the screen by a thin layer of collagenous tissue (fibrocellular proliferation), which extended from the submesothelial connective tissue deep into the external elastic lamina (fig 1). The submesothelial screen compartment could be stripped easily from the submesothelial adipose tissue compartment, presumably because the anatomical compartments of the pleura were not destroyed by the original inflammatory process and because the internal elastic was not affected. ${ }^{16}$ The density of the mononuclear infiltrate in a band-like pattern on the submesothelial adipose tissue compartment allows the recognition of the border between the submesothelial screen compartment and the submesothelial adipose tissue compartment.

In the third pattern of pleural involvement, apart from improving the morphological characterisation of chronic non-specific pleuritis or benign non-tuberculous pleuritis, the use of quantitative information led to the identification of patients who should continue to be under diagnostic investigation.

A normal pattern of pleural involvement was found in the fourth group of patients with clinical conditions associated with transudative effusions. However, $5.2 \%$ of patients with malignancies fell into this group. Therefore, the finding of a normal pleural pattern and a transudate does not exclude the diagnosis of malignancy.

We concluded that morphometric analysis of pleural biopsies could be useful as a supplementary histological procedure in the diagnosis of pleural tuberculosis and malignant disease. Because of its simplicity, efficiency, and low cost the use of morphometric tools in the routine diagnosis of pleural biopsies should be encouraged in the management of patients with pleural effusions.

This paper was presented in part at the 1996 International Chis paper was presented in part at the 1996 International May 10-15 and the 86th Annual Meeting of the United States and Canadian Academy of Pathology, Orlando, March 1-7, and $\mathrm{C}$.

This work was supported by the following Brazilian funding agencies: FAPESP, CAPES, CNPq, and LIM-HCFMUSP. We are grateful to Eugenia Deheinzelin for her help in the preparation of this manuscript.

1 Abrams LD. A pleural punch biopsy. Lancet 1958;i:30-1.

2 Cope C. New pleural biopsy needle: preliminary study. fAMA 1958;167:1107-8.

3 Donohoe RF, Katz S, Matthews MJ. Pleural biopsy as an aid in the etiologic diagnosis of pleural effusion: review of the literature and report of 132 biopsies. Ann Intern Med 1958; 48:334-62.

4 Levine $\mathrm{H}$, Cugell DW. Blunt-end needle biopsy of the pleura and rib. Arch Intern Med 1962;109:516-25.

5 Prakash UBS, Reiman HM. Comparison of needle biopsy Prakash UBS, Reiman HM. Comparison of needle biopsy
with cytologic analysis for the evaluation of pleural effusion: analysis of 414 cases. Mayo Clin Proc 1985;60 158-64.

6 Sahn SA. The pleura. Am Rev Respir Dis 1988;138:184-234.

7 Poe RH, Israel RH, Utell MJ, Hall WJ, Greenblatt DW, Kallay MC. Sensitivity, specificity, and predictive values of closed pleural biopsy. Arch Intern Med 1984;144:325-8.

8 Leslie WK, Kinasewitz GT. Clinical characteristics of the patient with nonspecific pleuritis. Chest 1985;94:603-8.

9 Nagata N, Kawarada Y, Shigematsu N, Ishibashi T Subpleural mononuclear cell infiltration: significance in the differential diagnosis of pleuritis showing nonspecific differential diagnosis of pleuritis showing

10 Von Hoff DD, LiVolsi V. Diagnostic reliability of needle biopsy of the parietal pleura: a review of 272 biopsies. $A \mathrm{~m}^{\mathcal{J}}$ Clin Pathol 1975;64:200-3.

11 Mestitz P, Purves MJ, Pollard AC. Pleural biopsy in the diagnosis of pleural effusion. Lancet 1958;ii:1349-53.

12 Gundersen HJG, Bendtesen TF, Korbo L, Marcussen N, Moller A, Nielsen K, et al. Some new, simple and efficient stereological methods and their use in pathological research and diagnosis. APMIS 1988;96:379-94.

13 PSS statistical software, version 6.0. Chicago: SPSS Inc, 1993.

14 Paterson RC. The pleural reaction to inoculation with tubercle bacilli in vaccinated and normal guinea pigs. $A m$ Rev Tuberc 1917;1:353-71.

15 Stead WE, Eichenholz A, Stauss H-K. Operative and pathologic findings in twenty-four patients with syndrome of idiopathic pleurisy with effusion presumably tuberculous. $A m$ Rev Tuberc 1955;71:413-502.

16 Hebert A. Pathogenesis of pleurisy, pleural fibrosis, and mesothelial proliferation. Thorax 1986;41:176-89.

17 Sahn SA. Malignant pleural effusions. Semin Respir Med 1987;9:43-53.

18 Stelzner TJ, King TE Jr, Antony VB, Sahn SA. The pleuropulmonary manifestation of the postcardiac injury synpulmonary manifestation of

19 Watanabe H, Enjoji M, Imai T. Gastric carcinoma with lymphoid stroma. Its morphologic characteristics and prognostic correlations. Cancer 1976;38:232-43. 